\title{
La innovación educativa en América Latina: lineamientos para la formulación de políticas públicas
}

\author{
Educational Innovations in Latin America: \\ guidelines for the formulations of public policies
}

\author{
Pablo Ríos-Cabrera \\ Docente, Universidad Pedagógica Experimental Libertador \\ Caracas, Venezuela \\ pablorioscabrera@gmail.com \\ Carlos Ruiz-Bolívar \\ Docente, Nova Southeastern University \\ Florida, Estados Unidos de América \\ cr1255@nova.edu
}

Recibido - Received: 29 / 02 / 2020

Corregido - Revised: 20 / 05 / 2020

Aceptado - Accepted: 27 / 05 / 2020

DOI: https://doi.org/10.22458/ie.v22i32.2828

URL: https://revistas.uned.ac.cr/index.php/innovaciones

\begin{abstract}
Resumen: El propósito del ensayo consiste en proponer un conjunto de lineamientos para la formulación de políticas públicas orientadas al desarrollo de innovaciones educativas en América Latina, a partir de la exploración de la literatura especializada, para determinar los aspectos críticos del desarrollo de innovaciones en el contexto latinoamericano. Se hizo una selección de los documentos en función de la credibilidad de las fuentes, actualidad y pertinencia de las referencias. En la primera parte del trabajo, se presenta una introducción sobre el problema del desfase en la relación entre sociedad y educación; se analizan también los factores que favorecen u obstaculizan la innovación educativa; la innovación educativa en América Latina y sus tendencias. En la segunda parte, se proponen los siguientes lineamientos: construir consensos, asumir una visión sistémica y contextualizada, valorar a los educadores, trabajar en comunidades de aprendizaje y aprovechar las tecnologías de información y comunicación. Se espera que estos lineamientos sirvan para: (a) formular políticas que orienten y favorezcan los cambios en educación; (b) planificar, desarrollar y evaluar las innovaciones de una manera más sistemática, eficiente y científica; y (c) contribuir a generar una cultura de la innovación educativa en América Latina.
\end{abstract}

Palabras clave: Innovación educativa, políticas públicas, educación latinoamericana, políticas educativas

Summary: The purpose of this work is to propose a set of guidelines for the formulation of public policies aimed at the development of educational innovations in Latin America, based on the exploration of specialized literature, to determine the critical aspects of the development of innovations in the context Latin American. A selection of the documents was made based on the credibility of the sources, news, and relevance of the references. In the first part of the paper an introduction is presented on the problem of the gap in the relationship between society and education; factors that favor or hinder educational innovation are also analyzed; educational innovation in Latin America and its trends. In the second part, the following guidelines are proposed: build consensus, assume a systemic and contextualized vision, value educators, work in learning communities and take advantage of information and communication technologies. It is hoped that these guidelines will serve to: (a) formulate policies that guide and favor changes in education; (b) plan, develop and evaluate innovations in a more systematic, efficient and scientific way; and (c) contribute to generate a culture of educational innovation in Latin America.

Key Words: Educational innovation, public policies, education, Latin American education, educational policies 
En teoría, la sociedad y la educación deberían mantener una relación de convergencia, donde los cambios que ocurren en la primera se vieran reflejados en la segunda. Esta relación funcionó bastante bien hasta mediado del siglo pasado en América Latina, particularmente porque la sociedad era bastante estable, cambiaba poco y en forma lenta. En la realidad presente, la sociedad se caracteriza por estar sometida a cambios rápidos, de orden científico-tecnológico y socio-culturales, a un proceso de globalización, y por el uso estratégico de la información y el conocimiento. Estas características de la sociedad del siglo XXI, exige con urgencia un tipo de educación que responda a las exigencias, el cual no se ha logrado consolidar todavía en América Latina, a pesar del esfuerzo que realizan algunas organizaciones y gobiernos de la región en esta dirección. En tal sentido, la UNESCO (2016) ha señalado que:

La urgencia de adecuar la educación a los cambios que vive la sociedad en el conocimiento, la tecnología, la información, los nuevos lenguajes, la comunicación y la investigación, llevó a incorporar a la innovación como aspecto central del nuevo escenario social. Ello ha incidido para que la innovación se convierta en una preocupación de la educación en la segunda mitad del siglo XX, siendo transferida desde el mundo de la administración y de la empresa. En consecuencia, se ha llegado a considerarla necesaria para lograr la modernización de una escuela que requiere adecuarse a los nuevos tiempos (p. 11).

Lo anterior plantea un gran reto para los gobiernos, líderes institucionales, planificadores y profesionales, en general, que se desempeñan en estas áreas; en sus manos está la búsqueda de soluciones alternativas que progresivamente contribuyan a cerrar la brecha entre las exigencias de la sociedad y la calidad y pertinencia de la educación.

El desfase educación-sociedad no se puede atender sobre la base de soluciones convencionales basadas en reformas o cambios parciales en la estructura del sistema educativo. Se requiere de una estrategia que permita reducir esta brecha a fin de encontrar un mayor equilibrio entre las demandas de la nueva sociedad y la calidad, pertinencia y equidad de oferta educativa. La estrategia no es otra que la innovación, la cual debe estar enfocada a cambiar estructuralmente el sistema educativo tradicional. Para tal fin, se hace necesaria la formulación de políticas públicas orientadas a crear las condiciones apropiadas a lo interior de los sistemas educativos a fin de garantizar un proceso de innovación exitoso que contribuya al mejoramiento del servicio educativo. En este sentido, el trabajo tiene como propósito proponer un conjunto de lineamientos para la formulación de políticas públicas para el desarrollo de la innovación educativa en América Latina.

El término innovación ha sido muy estudiado y aplicado inicialmente $y$, a través del tiempo, en las organizaciones productivas. En este sentido, Gros y Lara (2009) definen la innovación como el proceso de creación de conocimientos, productos y procesos nuevos, el cual conforma una parte esencial del trabajo de las organizaciones ya que es un valor imprescindible para la sociedad del siglo XXI. Sin embargo, para algunos autores (por ejemplo, Senge, 2011), la innovación no está dirigida solo a mejorar los procesos y productos, sino que también orientada a modificar, de manera sustantiva, las creencias, hábitos, valores, actitudes y tradiciones entre los grupos existentes en todas las organizaciones, es lo que en este contexto se denomina cultura organizacional (Shein, 2012, Banerjee, 2017).

De acuerdo con Senge (2011), la innovación estaría dirigida a cambiar los modelos mentales de las personas, entendidos como estructuras cognitivas inconscientes de los individuos que determinan su modo de comprender el mundo, de actuar y de sentir; pueden ser generalizaciones, supuestos hondamente arraigados, teorías, imágenes o paradigmas. 
Estas ideas básicas sobre la palabra innovación, nos permiten avanzar en una reflexión de fondo sobre la aplicación de dicho término en el contexto educativo y ello lleva a preguntarse, en principio, ¿qué es una innovación educativa? Para responder a la interrogante se seleccionaron tres definiciones que representan diferentes enfoques sobre el tema, como se indica a continuación:

UNESCO (2014) entiende la innovación educativa como "un acto deliberado y planificado de solución de problemas, que apunta a lograr mayor calidad en los aprendizajes de los estudiantes, superando el paradigma tradicional. Implica trascender el conocimiento academicista y pasar del aprendizaje pasivo del estudiante a una concepción donde el aprendizaje es interacción y se construye entre todos" (p. 3).

Gros y Lara (2009) definen a la innovación como "el proceso de creación de conocimientos, productos y procesos nuevos, el cual conforma una parte esencial del trabajo de las organizaciones ya que es un valor imprescindible para la sociedad del siglo XXI" (p. 225).

Carbonell (2002), entiende la innovación educativa como: "(un) conjunto de ideas, procesos y estrategias, más o menos sistematizados, mediante los cuales se trata de introducir y provocar cambios en las prácticas educativas vigentes" (pp. 11-12).

Del análisis de las definiciones presentadas por los autores precitados, se destacan las ideas siguientes: (a) no existe consenso entre los especialistas acerca de lo que se entiende por innovación educativa; (b) la innovación educativa, como un proceso de cambio planeado y deliberado, transformador de la realidad educativa, que debe ser adaptativa y estar en sintonía con las necesidades propias del contexto educativo y de la sociedad; (c) la innovación como creación de conocimientos, productos y procesos nuevos; y (d) tiene como propósito el mejoramiento de la calidad del aprendizaje y de la educación, con equidad (Murillo 2017, p. 1).

Desde un punto de vista teórico, los autores entienden la innovación educativa como un proceso complejo dirigido a transformar los diferentes factores que intervienen en el aprendizaje, como son: la concepción, estructura y contenido curricular, las prácticas pedagógicas tradicionales de los docentes, la responsabilidad de los estudiantes frente a su aprendizaje, la calidad y pertinencia de los recursos educativos, las técnicas de evaluación y la gerencia del plantel, entre otros. Es importante señalar que no se trata de una sumatoria de factores que actúan de manera individual o independiente, o de la mejora de un aspecto en particular, sino de una acción conjunta, interactiva y complementaria entre dichos factores para lograr el objetivo de una educación de calidad, pertinente y equitativa. Es decir, se refiere a un tipo de innovación disruptiva que tiene por objeto impactar todo el contexto educativo.

Este tipo de innovación permite "que la evolución lineal de un método, técnica o proceso de enseñanzaaprendizaje cambien drásticamente alterando la evolución lineal del contexto educativo, modificando permanentemente la forma en la que se relacionan los actores del contexto, los medios y el entorno mismo" (Murillo 2017, p. 1).

Esta visión de la innovación educativa es consistente con la formulación de Morín (2000) sobre la teoría del pensamiento complejo. Desde esta perspectiva, la realidad se concibe como un todo formado por constituyentes heterogéneos, inseparablemente asociados. Él considera que "la complejidad es efectivamente el tejido de eventos, acciones, interacciones, retroacciones, determinaciones y azares que constituyen nuestro mundo fenoménico" (p. 32). 


\section{FACTORES QUE FAVORECEN U OBSTACULIZAN LA INNOVACIÓN EDUCATIVA}

Existe consenso entre los diferentes especialistas en que innovar en educación no es una tarea fácil, por el contrario, es bastante compleja, y así como tiene un conjunto de factores que la favorecen, también supone el reto de superar obstáculos personales, institucionales o socioculturales que se asocian al proceso de cambio transformador que la innovación implica. A continuación, se presentan organizados en tres niveles algunos factores que posibilitan el éxito en un proceso de innovación educativa.

1. A nivel global del sistema educativo, la formulación e instrumentación práctica de políticas públicas específicas y apropiadas son indispensable para direccionar y guiar la acción innovadora a nivel de los planteles o centros educativos y su implementación en espacios educativos. Asimismo, son imprescindibles los aportes financieros que se requieren para cubrir los costos que toda innovación educativa supone, por ejemplo, en dotación de equipos, adquisición de material didáctico, entrenamiento de docentes, asesoramiento para el rediseño curricular y mantenimiento de centros, entre otros.

2. En el contexto del plantel o centro educativo, es deseable contar con una gerencia comprometida con el cambio, que lidere y anime a la comunidad educativa a involucrarse en el proceso de innovación a través del trabajo en equipo, que fomente un clima organizacional positivo y promueva un sistema de evaluación continua del proceso que permita retroalimentar los cambios y tomar decisiones oportunas.

3. A nivel de los espacios educativos es necesario contar con un curriculum actualizado, como parte de la innovación, un personal docente consciente de los objetivos del proceso de innovación, que promueva la autonomía y la responsabilidad del estudiante en su aprendizaje, capacitado en el uso de nuevos métodos instruccionales, especialmente aquellos que incorporan explícitamente el recurso de las tecnologías de la información y la comunicación en el proceso.

En cuanto a los obstáculos, es importante estar consciente que en todo proceso de innovación no todos los actores están igualmente motivados para participar activamente en el proceso, mostrando comportamientos evidentes de resistencia al cambio, entendida como "el levantamiento de barreras por temor a lo desconocido, por desconfianza hacia los indicadores de cambio o por sentimientos de seguridad amenazada" (Núñez de Sarmiento y Gómez, 2005, p. 27). Esta situación ha sido ampliamente estudiada en la psicología del trabajo, en el contexto de las organizaciones productivas. Se trata de un fenómeno psicológico global que está presente, en mayor o menor grado, en todas las personas; afecta el éxito de las iniciativas de cambio que emprenden las organizaciones, y tiene la capacidad de socavar seriamente las mismas y conducir al fracaso de los proyectos de innovación impulsados desde la Gerencia (Stewart, McCarthy, May y Puffer, 2009).

\section{LA INNOVACIÓN EDUCATIVA EN AMÉRICA LATINA}

En esta sección se analizan los principales hitos de la innovación educativa en América Latina. Para algunos autores (UNESCO, 2016; Barraza, 2005; Blanco y Messina, 2000, entre otros), el proceso se inició en la década de los años 60, vinculado a las ciencias administrativas, como sinónimo de modernización. La innovación estaba asociada a los programas de reformas educativas que se implantaron para la época; fue concebida como un proceso externo dirigido por expertos, particularmente por los planificadores, quienes lo consideraban como un programa de cambio planeado y sistemático. Este tipo de innovación impuesta desde afuera no llegó a tener el impacto esperado en cuanto a la transformación de la cultura escolar. 
Blanco y Messina (2000) consideran que durante la décadas de los años 70 y 80, la innovación educativa fue liderada por los propios docentes. Se produjo un gran desarrollo de experiencias innovadoras en la educación formal, no formal y comunitaria. "La mayoría de las experiencias se nutrieron de diferentes corrientes pedagógicas que denunciaban desde la práctica, una educación academicista que no promovía el pensamiento crítico, ni la autonomía y no proporcionaba herramientas para transformar la realidad" (p. 42). Ellas agregan que en la década de los 90 la innovación se enfocó en la transformación global de los sistemas educativos con el propósito de mejorar la equidad y la calidad de la educación, así como la eficacia y eficiencia del sistema educativo; las innovaciones estuvieron asociadas a modelos de descentralización y autonomía de los centros educativos.

Para Fullan (2002), de la década de los 90 en adelante las innovaciones se han centrado en capacitar para el cambio. Considera que "no podemos depender o esperar a que el sistema cambie (p. 75)", ya que los sistemas educativos son intrínsecamente no lineales y endémicamente fragmentados e incoherentes. Características también presentes en las sociedades complejas. De allí concluye que "debemos desarrollar nuestras propias capacidades individuales para aprender y para seguir aprendiendo sin dejar que nos derrumben las vicisitudes del cambio (p. 75)", así como crear patrones coherentes en los individuos y en los grupos.

En el presente, la UNESCO $(2016$, p. 11) ha planteado que la innovación está fundamentada sobre el aprendizaje, en cuanto este se encuentra ligado a la acción transformadora del mundo. Tiene un profundo sentido de cambio pues produce unas características que no se dan por generación espontánea. Estas deben ser organizadas y planificadas para que el espacio de innovación-aprendizaje logre sus impactos en los múltiples ámbitos de la sociedad. Asimismo, plantea que en el siglo XXI la innovación se ha vinculado, por una parte, con la incorporación de las nuevas tecnologías de la información y la comunicación (TIC) en la educación y, por otra, con el protagonismo de las instituciones educativas.

\section{TENDENCIAS ACTUALES DE LA INNOVACIÓN EDUCATIVA EN AMÉRICA LATINA}

Londoño (2017), con base en un estudio del Banco Interamericano de Desarrollo (2014), identificó nueve tendencias en la innovación educativa en América Latina, las cuales se sintetizan en este apartado:

1. Sistemas de Ensino. Es un modelo de enseñanza iniciado en Brasil que utiliza un conjunto de dispositivos integrales de apoyo pedagógico; ofrecen: libros de texto, portales educativos de acceso exclusivo, sistemas de gestión, foros y web 2.0 para el uso exclusivo de docentes, padres y alumnos (p. 51).

2. Uso de videos educativos. El uso de videos instruccionales de corta duración ha sido empleado extensivamente por la Academia Khan (Khan, 2019) en su programación y gestión de cursos virtuales gratuitos para estudiantes con diferentes necesidades educativas. Progresivamente, su uso se ha ido extendiendo internacionalmente, aplicados en diferentes modalidades de cursos de auto-instrucción, semi-presenciales, de la clase invertida en el aprendizaje móvil o incluso para apoyar el aprendizaje de alumnos en las clases presenciales (p. 51).

3. Portales de materiales educativos y recursos abiertos. Londoño señala que estos portales diversifican la oferta de contenidos educativos. Dan propuestas a docentes y alumnos y abren un tiempo de aprendizaje fuera del aula, brindando así una amplia gama de posibilidades diversas para la planificación didáctica (p. 51).

4. Redes de escuelas, docentes y alumnos. Aprovechando los recursos de las tecnologías y de internet, se observa que algunas escuelas se organizan en redes y participan a través de diferentes plataforma 
que facilitan las interacciones entre los docentes y sus alumnos; un ejemplo de ello lo constituye la plataforma Edoome, la cual provee un entorno familiar y sencillo, donde los profesores pueden continuar las discusiones de sus clases con herramientas que hacen sencilla la forma de compartir nuevo contenido, tópicos de discusión o resolver tareas en grupo (p. 51).

5. Nuevos programas educativos contra la pobreza. Están referidos a proyectos de innovación que combinan el uso de las tecnologías y la creación de escuelas con nuevos modelos pedagógicos en contextos vulnerables, con el apoyo de grupos privados. Algunas experiencias de este tipo han sido implementadas en países como Chile con el proyecto Puentes Educativos, o en Colombia con el programa Transformemos, y también en Panamá en el Instituto de Educación por Radio, entre otros (p. 51).

6. La renovación de las ciencias. Se trata de un nuevo enfoque en la enseñanza de las ciencias a partir del uso de los videojuegos, entre otros recursos. Es decir, el proyecto está enfocado a la renovación de las áreas tradicionales de enseñanza a partir de nuevos materiales, tecnologías y enfoques instruccionales novedosos. Un ejemplo de esta tendencia son las siguientes dos iniciativas: Avatar y Kokori (p. 51).

7. Escuelas innovadoras con tecnologías. Consiste en el uso intensivo de tecnologías en escuelas públicas, privadas o mixtas orientadas con un enfoque de proyectos de escuelas innovadoras. "Estas escuelas, están cambiando el imaginario educativo, están desplegando nuevas formas de habitar las escuelas, cambiando las arquitecturas y creando una infraestructura digital (p. 52)". Una muestra de ello lo constituye el proyecto Innova Schools de Perú.

8. El aprendizaje personalizado por computadores. Esta tendencia está basada en la personalización del aprendizaje asistido por computadoras. Un caso avanzado es el de Geekie en Brasil, un sitio web a través del cual se busca mejorar las competencias de los estudiantes por medio de resoluciones de exámenes, participación en juegos lógicos y la atención virtual personalizada (p. 52)".

9. Movimientos educativos virales y ciudadanos no tradicionales. Esta tendencia agrupa movimientos educativos virales no tradicionales, campañas masivas, series, movimientos de cambio, de interrogantes, de incidencia política, de ideas. Un ejemplo, en esta tendencia son los proyectos TEDxRíodelaPlata en Argentina, El Reemplazante, en Chile y Un millón de jóvenes por México (p. 52).

\section{LINEAMIENTOS PARA LA FORMULACIÓN DE POLÍTICAS PÚBLICAS}

En este apartado los autores de este artículo proponen un conjunto de lineamientos para la formulación de políticas públicas y praxis pedagógicas orientadas a la promoción y desarrollo de innovaciones educativas que de alguna manera superen los factores adversos antes indicados. Se consideraron cinco aspectos: (a) construir consensos; (b) asumir una visión sistémica y contextualizada; (c) valorar a los educadores; (d) trabajar en comunidades de aprendizaje; y (e) aprovechar las tecnologías de información y comunicación.

\section{Construir consensos}

Las políticas públicas son el ámbito privilegiado de realización del "pacto" entre el Estado y la sociedad. A principios de los 90 crece la convicción de que la transformación educativa sólo sería posible con el apoyo consensuado y el compromiso de todos los sectores sociales. La declaración de la Conferencia Mundial sobre Educación para Todos (UNESCO, 1990) recogió explícitamente este aspecto: "Será necesaria la concertación de acciones nuevas y revitalizadoras a todos los niveles" (p. 12), apelando a la concertación entre "organizaciones gubernamentales y no gubernamentales, el sector privado, las comunidades locales, los grupos religiosos y las familias" (p. 13). 
Estos procesos pueden tener diferentes denominaciones como: concertaciones, acuerdos, pactos, mesas de diálogo, consensos educativos, etc. Los pactos son acuerdos consensuados socialmente a través de procesos de concertación. Las dificultades para concertar políticas educativas vienen dadas porque la información y el conocimiento constituyen cada vez más las variables claves de la distribución del poder, de allí que el control de su producción y distribución se convierte en el ámbito de los conflictos sociales más significativos (Tedesco, 2005).

El valor de los pactos no se debe evaluar sólo en función de su resultado final, sino también por el proceso de debate, negociación, aprobación y ejecución que promueven, ya que los actores sociales deben acordar normas transparentes, definidas en el espacio público así como discutir y negociar abiertamente sus opciones educativas. Además, deben llegar a consensos sobre las reglas de juego a través de las cuales se van a dirimir las diferencias y los desacuerdos, de tal forma que no es una sola y única visión que todos deben asumir, ni muchas y cada uno con la suya, sino que es una consensuada, construida con las de todos.

El pacto no es un instrumento válido en sí mismo, sino que constituye un procedimiento consistente con un proyecto social basado en la idea de construir una sociedad libre y democrática. Para Tedesco (op. cit.), "concertar políticas educativas es parte de un proceso más general de fortalecimiento de la ciudadanía y de construcción de un orden político democrático" (p. 27). En definitiva, han de ser políticas construidas de manera participativa y de largo plazo, con permanencia y continuidad más allá de cada gobierno y de las prioridades particulares de cada sector, llegando así a convertirse en políticas de Estado.

\section{Asumir una visión sistémica y contextualizada}

Habitualmente, se tiene una visión fragmentada y parcial de los fenómenos lo cual impide captar la totalidad, comprender la complejidad, multicausalidad e interdependencia, a la cual aspira el conocimiento científico y la acción social y política eficaz (Torres, 2005). Un sistema es un conjunto de partes o elementos organizados y relacionados, que interactúan entre en sí para lograr determinados objetivos. Lo educativo ha de entenderse de manera sistémica e integral, en sus diversos componentes: pedagógico, curricular, administrativo, actores: alumnos, familias, comunidad, docentes, directivos, asesores y funcionarios, instancias nacionales y organismos internacionales y ámbitos: local, nacional, regional e internacional (Torres, 2005).

Igualmente, para Fullan (2002), los cambios educativos tienen que ser sistémicos, es decir, abarcar no sólo las estructuras formales, sino a los actores, a los procesos académicos y administrativos e incidir en nuevas formas de relación, pensamiento y participación. Hay que cuidar la coherencia que los diversos elementos puedan tener entre sí, como aspecto que guía y evidencia la relación y congruencia de todos los elementos del hecho educativo. En definitiva, ha de ser un cambio sistémico de la organización educativa en su conjunto ya que la incorporación de cambios en algún componente tiene repercusiones en otros con los que se relaciona e interactúa.

\section{Valorar a los educadores}

El educador es el actor fundamental para la instrumentación de cualquier cambio en los procesos educativos. Aunque las políticas públicas o las innovaciones educativas estén concebidas de la mejor manera posible, ello de ninguna manera suplanta la capacidad ni la voluntad de los individuos encargados de llevarlas a la práctica (BID, 2018). Al respecto consideraremos la importancia de los educadores y su capacitación. 
La revisión de la literatura evidencia que los desafíos a los que se enfrentan las sociedades y las economías ubican a la educación en el centro del debate y a los docentes como actores clave (Vaillant, 2006). Los educadores son cada vez más reconocidos como los actores principales en los esfuerzos por alcanzar mejores resultados en la calidad de la educación. Del estudio de los sistemas educativos exitosos, Barber y Mourshed (2008), señalan: "Los sistemas con más alto desempeño demuestran que la calidad de un sistema educativo depende en última instancia de la calidad de sus docentes" (p. 39). Resaltan que estos sistemas le dan importancia a tres aspectos, a saber: (a) conseguir a las personas más aptas para ejercer la docencia; (b) formarlos con altos estándares académicos y enfoques innovadores hasta convertirlas en educadores eficientes; $y$ (c) mejorar las condiciones de vida y trabajo a fin de que se mantengan en el sistema educativo.

El informe citado indica que estos objetivos pueden alcanzarse independientemente del contexto y la cultura, lograrse en el corto plazo y mejorar las dificultades de los sistemas educativos en cualquier lugar del mundo (Castillo, 2013). Para Barber y Mourshed (2008) "la evidencia disponible sugiere que el principal impulsor de las variaciones en el aprendizaje escolar es la calidad de los docentes" (p. 12), son sistemas educativos que apuestan fuertemente a la mejora de la instrucción. Esto implica mejorar la interacción entre alumnos y docentes, mejorar las estrategias didácticas, llevar la capacitación docente a los espacios educativos, desarrollar líderes con mayores capacidades y facilitar la retroalimentación entre docentes.

En UNESCO/OREALC (2007), se reconoce que "Ios docentes deben trabajar en nuevos escenarios, para los cuales no han sido ni están siendo suficientemente preparados" (p. 49), por tanto, se debe avanzar en su profesionalización. Ahora bien, todo profesional necesita estar actualizado en su campo de desempeño y la profesión docente no escapa al requerimiento. Para Aguerrondo (2009), "un profesional no se hace durante sus estudios, sino fundamentalmente cuando empieza a trabajar" (p. 368). Ese recorrido desde el conocimiento académico a la acción es lo que lo constituye como profesional. Los docentes tienen que actualizar y profundizar sus conocimientos de manera permanente como forma de dar respuesta a los retos de una época de incertidumbres y a sociedades cada vez más complejas, globalizadas y cambiantes.

Pero, como se señala en UNESCO/OREALC (2007): "La calidad de la formación de los docentes es uno de los nudos críticos de los sistemas educativos" (p. 52), este problema abarca tanto a los procesos de formación inicial como a los de formación continua o en servicio. De acuerdo con Barber y Mourshed (2008), hay tres principios clave detrás de los sistemas educativos con muy buenos desempeños:

1. La calidad de un sistema educativo no puede ser superior a la calidad de sus docentes.

2. El único camino para mejorar los aprendizajes es mejorar la enseñanza.

3. Un buen desempeño como sociedad requiere que todos los estudiantes aprendan.

Sin embargo, frecuentemente ocurre que "en las escuelas públicas, los docentes provienen crecientemente de sectores pobres de la población, poseen una educación muy deficitaria, algunos tienen habilidades básicas limitadas, reciben escasa formación y perciben bajas remuneraciones" (Hunt, 2008, p. 6). Por otra parte, la educación que imparten es tradicional, centrada básicamente en la transmisión de información, la memorización de contenidos, con una actitud pasiva frente al cambio y las innovaciones educativas; donde se trabaja de forma más individual que colaborativa. Según UNESCO/ OREALC (2012): 
A pesar de la intensa actividad que se registra a lo largo de las últimas tres décadas en América Latina y El Caribe en torno a la formación y capacitación de los docentes, los análisis revelan su bajo impacto en el aula y evidencian una opinión adversa de muchos directores y a veces de los propios profesores sobre los cursos, talleres, programas compensatorios y del vasto conjunto de esfuerzos que, más allá de las intenciones, no han correspondido a las expectativas de mejora de las prácticas educativas necesarias para incrementar de manera sostenida los logros de todos los estudiantes (p. 58).

Igualmente señala: "Se ha demostrado que el formato de cursos ha tenido debilidades notables tales como la duración limitada, la distancia entre sus contenidos y los requerimientos de las escuelas, y la falta de seguimiento de la aplicación en la práctica" (p. 58). En el mismo sentido, Hunt (2008) indica que "con frecuencia consisten en talleres breves, que brindan poca oportunidad y escaso apoyo para aplicar metodologías nuevas en sus aulas" (p. 7).

\section{Trabajar en comunidades de aprendizaje}

Frecuentemente, las problemáticas, tanto de la praxis pedagógica como del contexto social y cultural de las instituciones educativas son de tal magnitud y complejidad que desbordan las capacidades individuales del personal docente, por lo que para afrontarlas la mejor opción es pensar y actuar en equipo, en redes, en clústers; sumando e integrando las competencias y habilidades individuales, para responder desde múltiples perspectivas. Ello favorece el surgimiento de nuevas ideas frente a una misma realidad, a la vez que se crean nexos significativos entre los docentes y entre estos y los directivos, donde se generan relaciones más horizontales y de complementariedad de enfoques, opiniones y puntos de vista hasta llegar a una definición compartida de los problemas por resolver, en la que todos los involucrados puedan aportar su perspectiva y análisis (Scardamalia y Bereiter, 2016).

Lo anterior también significa valorar la diversidad como algo que enriquece a las personas e instituciones educativas. De allí que la innovación no se emprende desde el aislamiento, la soledad y la fragmentación, sino desde el intercambio profesional y la cooperación permanente como fuente de contraste y enriquecimiento mediante la creación de redes o colectivos intra e interinstitucionales (Barraza, op. cit.). Esta relación de colaboración también ha de darse con las familias y entre los propios estudiantes.

Uno de los temas emergentes que encontraron Blanco y Messina (2000), fue un movimiento interesante hacia la colaboración, a través de redes, entre escuelas y profesores para debatir aspectos pedagógicos e intercambiar experiencias. Igualmente, el Banco Mundial (2018) destaca la importancia de construir alianzas y coordinar a los actores para que todo el sistema favorezca el aprendizaje mediante redes de intercambio, espacios de encuentro y publicaciones compartidas.

Según Escudero (2009), las comunidades de aprendizaje son una modalidad de formación, capacitación y acompañamiento donde grupos de personas y sus instituciones, con un interés común de aprender y hacer más efectivas sus prácticas, desarrollan mecanismos para compartir experiencias y conocimientos que, a través de la interacción sostenida y cooperativa, apuntan a la innovación, el desarrollo de capacidades y el mejoramiento de las prácticas. Se sustentan en metodologías basadas en la resolución de problemas de forma colectiva, integrando diversos puntos de vista en su solución y favoreciendo la creatividad y la innovación. Se basan en la confianza y en el reconocimiento de la diversidad y surgen en áreas con problemas complejos. Comparten ideas y recursos de aprendizaje y colaboran en la realización de proyectos comunes. En definitiva, las comunidades o redes de aprendizaje representan una alternativa organizada para el aprendizaje continuado y el desarrollo de innovaciones. 


\section{Aprovechar las tecnologías de la información y la comunicación}

Los descubrimientos científicos y las innovaciones tecnológicas tienen un profundo impacto en el desarrollo de la humanidad, así lo certifican las invenciones de la escritura, la imprenta, el teléfono, la radio, el cine o la televisión. La expresión tecnologías de la información y la comunicación (TIC), se refiere a toda la gama de servicios, aplicaciones y tecnologías que utilizan tanto equipos (hardware) como programas informáticos (software) que habitualmente se transmiten a través de redes de telecomunicaciones (netware) (Sánchez, 2016). En estas tecnologías se integran conocimientos de la informática, la microelectrónica y las telecomunicaciones y nos permiten buscar, almacenar, procesar, administrar, recuperar, producir y difundir todo tipo de contenidos mediante diferentes soportes tecnológicos, a través de símbolos de naturaleza acústica, óptica o electromagnética (Briones, 2001). Son tecnologías con un papel fundamental en la configuración de nuestra sociedad y nuestra cultura, con impacto en todas las actividades, en todos los países y en todos los individuos.

Entre las características básicas de la sociedad de la información están la elaboración de conocimientos en red, los intercambios donde se superan las barreras del tiempo y del espacio, la internacionalización, el cambio y la proliferación de los flujos de datos, información, conocimientos, experiencias, personas, productos y servicios, todo lo cual influye en una total reestructuración del sistema educativo, permitiendo a los individuos autorrealizarse en ambientes caracterizados por los cambios sociales, económicos y tecnológicos.

Como ocurre en muchos otros ámbitos, el uso de las TIC en educación tiene tanto defensores como detractores. Así, para Briones (2001), la tecnofilia se refiere a la completa confianza en las tecnologías, a exaltar sus dimensiones positivas y a verlas como "la solución a los problemas educativos" (p. 71). Se plantea la necesidad de una infraestructura digital que posibilite un salto en la generación de conocimientos; o que la incorporación de tecnología en el sistema educativo garantice una mejor calidad de la educación. Entre las ventajas hallamos que facilitan la comunicación a larga distancia, brindan acceso a información abundante y variada, permiten el desarrollo de actividades u operaciones a través de la red, dan lugar a la creación de nuevos empleos en el área de telecomunicaciones y favorecen el impulso de los negocios y la actividad empresarial.

Para Brunner (2003), entre las potencialidades de las TIC en educación están el posibilitar una mejor visualización de procedimientos abstractos, facilitar la labor diagnóstica mediante evaluaciones más frecuentes y sofisticados, ayudar en las tareas de recuperación de los aprendices con problemas y rezagados, servir de apoyo a la memoria, permitir viajar a través del tiempo, ofrecer la posibilidad de que los estudiantes repitan las actividades a su propio ritmo, mejorar la motivación de los estudiantes mediante el diseño de programas personalizados, fomentar el trabajo en equipos, etc.

Por el contrario, desde la tecnofobia se rechaza o se desconfía de las posibilidades que ofrecen las tecnologías, se destacan los "efectos deshumanizadores y las conspiraciones comerciales, como una amenaza para la democracia y la formación de las nuevas generaciones" (p. 71). Se consideran peligrosas en la medida en que pueden eliminar puestos de trabajo, tradiciones, las posibilidades de interactuar cara a cara, e incluso nuestra propia conciencia, transformándonos en engranajes manejados por intereses económicos y políticos.

Entre las promesas o expectativas fundamentales asociadas al uso de las TIC en educación hallamos las siguientes:

a) Que las generaciones jóvenes adquieran destrezas y competencias basadas en estas tecnologías para integrarse a una sociedad crecientemente organizada en torno a ellas, a lo que comúnmente se llama alfabetización digital; 
b) que se reduzca la brecha digital entre individuos y grupos sociales, al propiciar acceso universal a computadores e internet;

c) que se mejoren las prácticas educativas y los logros académicos de los estudiantes mediante cambios en las estrategias de enseñanza y aprendizaje (Area, 2011).

Como bien señala Lugo (2010), "Ias TIC no son la panacea para los problemas educativos, sino una ventana de oportunidad para innovar en la gestión del conocimiento, en las estrategias de enseñanza, en las configuraciones institucionales, en los roles de los profesores y los alumnos" (p. 52).

Estas tecnologías ofrecen un gran potencial para impulsar el desarrollo social sustentable a través de la educación, para lo cual se hace necesario que el propósito de la innovación apoyada en las TIC transcienda el ámbito educativo "per se" y se conciba en el contexto de la realidad social latinoamericana a fin de que sus resultados contribuyan también a cerrar la actual brecha socioeconómica, educativo-cultural y tecnológica existente entre los países, en el marco de la propuesta de UNESCO (2000) sobre la educación para todos. En este sentido ellos han planteado lo siguiente:

Las tecnologías de la información y la comunicación deben ponerse en práctica de modo tal que sean un apoyo para alcanzar los objetivos de la EPT [Educación para Todos] a un costo razonable. Si bien estas tecnologías tienen un gran potencial para divulgar conocimiento, fomentar un aprendizaje más efectivo y desarrollar servicios educativos más eficientes, este potencial no podrá explotarse al máximo a menos que las tecnologías estén al servicio de la puesta en marcha de estrategias educativas, y no a la inversa. Para ser efectivas, especialmente en los países en desarrollo, las TIC deben combinarse con métodos más tradicionales, como el uso de libros y radios y aplicarse en forma más extensiva a la formación docente. (Prólogo)

Cuando se habla de la necesidad de incorporar las TIC como un recurso para mejorar la calidad, equidad y pertinencia de la educación la literatura especializada señala que la incorporación, por sí misma, como ya se señaló, no mejoran la calidad de la educación (ver, por ejemplo, Coll y Monereo, 2008; OEI-IESME, 2018; Dávila, Ruiz-Bolívar y Francisco, 2013). Para lograr el propósito se requiere de un proceso de innovación educativa donde la integración de estas tecnologías suponga una verdadera transformación del sistema cuyo efecto tenga un impacto sustantivo en cada uno de sus componentes, a fin de lograr el perfil de un ciudadano suficientemente equipado para aprender en forma permanente y vivir en democracia, de acuerdo con las exigencias de la sociedad del siglo XXI.

\section{CONCLUSIONES}

En el trabajo se constató que, aun cuando las innovaciones educativas pueden constituirse en un factor fundamental para mejorar la calidad de la educación, su efectividad está asociada a importantes complejidades y dificultades que, particularmente en el contexto latinoamericano, hay que revisar detenidamente. A partir del análisis de esta problemática, se propuso un conjunto de lineamientos para la formulación de políticas públicas orientadas al desarrollo de innovaciones en educación.

Como punto de partida, se consideró la necesidad de construir consensos con la participación de los actores fundamentales de la sociedad, a fin de que dichas políticas tengan relevancia, difusión y sostenibilidad (Anderson, 2016), y que, idealmente, puedan convertirse en políticas de Estado.

Como segundo lineamiento, se planteó la importancia de asumir una visión sistémica y contextualizada, debido a que, aun cuando se tengan pautas generales y metas comunes por lograr para el sistema, cada institución tiene peculiaridades que no se pueden uniformar. El éxito de una innovación en un contexto no garantiza que funcione en otro. 
Como tercer aspecto se consideró fundamental valorar a los educadores como actores clave para el desarrollo de las innovaciones educativas. Ello implica que cuenten con las condiciones y recursos necesarios, con una adecuada formación inicial, capacitación en servicio, asesoría técnica, acompañamiento y supervisión y todo aquello que contribuya a su desarrollo profesional.

Como forma de potenciar la labor del docente al llevar adelante innovaciones educativas se estimó necesario trabajar en comunidades de aprendizaje con lo cual se puede generar conocimiento y aprender a partir de los problemas cotidianos, afrontados en conjunto con otros colegas, así como actuar colectivamente de forma reflexiva, profesional y dialógica hacia el pleno desarrollo de todas las potencialidades de los estudiantes.

Por último, se analizó la importancia de aprovechar las tecnologías de información y comunicación, las cuales constituyen un factor clave en la mayor parte de las innovaciones educativas en la actualidad; el reto es superar la simple dotación de equipos que frecuentemente se usan para una educación tradicional y utilizar sus potencialidades pedagógicas, lo cual conlleve a cambios necesarios en las estrategias de aprendizaje y así mejorar los logros académicos de los estudiantes.

Esperamos que este trabajo constituya un aporte para superar el habitual desfase que existe entre las demandas de las sociedades actuales y los ritmos de cambio del sistema educativo y que sirva para propiciar una cultura de innovación en educación. Después de todo, estos lineamientos son sugerencias, propuestas de acción, con la aspiración de que se vayan instrumentando, desarrollando y evaluando en la práctica.

\section{REFERENCIAS}

Aguerrondo, I. (2009). Formación docente en la sociedad del conocimiento. Revista Estudos em Avaliacão Educacional. Fundacão Carlos Chagas, Rio de Janeiro, 363-387.

Anderson, S. (2016). Llevando innovaciones en programas educativos a gran escala: perspectivas, estrategias y desafíos. En Cueto, S. (Ed.). Innovación y calidad en educación en América Latina. Iniciativa Latinoamericana de Investigación para las Políticas Públicas (ILAIPP) Grupo de Análisis para el Desarrollo (GRADE), Lima, Perú.

Area, M. (2011). Los efectos del modelo 1:1 en el cambio educativo en las escuelas. Evidencias y desafíos para las políticas iberoamericanas. En Revista Iberoamericana de Educación 56, 49-74. Recuperado de https://rieoei.org/historico/documentos/rie56a02.pdf

BID (Banco Interamericano de Desarrollo). (2018). Profesión: profesor en América Latina. ¿Por qué se perdió el prestigio docente y cómo recuperarlo?. Washington, D.C. Recuperado de https://publications. iadb.org/handle/11319/8953

Banco Mundial (2018), Informe sobre el desarrollo mundial 2018: Aprender para hacer realidad la promesa de la educación, cuadernillo del "Panorama general". Banco Mundial, Washington, DC.

Banerjee, B. (2017). Why innovate? En: Banerjee, B., \& Ceri, S. (Eds.). Creating Innovation Leaders. A Global Perspective. Switzerland: Springer International Publishing.

Barber, M. y Mourshed, M. (2008). Cómo hicieron los sistemas educativos con mejor desempeño del mundo para alcanzar sus objetivos. Santiago de Chile: PREAL.

Barraza, A. (2005). Una conceptualización comprehensiva de la innovación educativa. Innovación Educativa, 5(28), 19-31. Recuperado de https://www.redalyc.org/articulo.oa?id=179421470003

Blanco, R. y Messina, G. (2000). Estado del arte sobre las innovaciones educativas en América Latina. Bogotá: Convenio Andrés Bello. 
Briones, S. (2001). Las tecnologías de la información y la comunicación: su impacto en la educación. PixelBit. Revista de Medios y Educación, 17, 67-78. Recuperado de https://idus.us.es/handle/11441/45527

Brunner, J. (2003). La educación al encuentro de las nuevas tecnologías. En J.J. Brunner y J.C. Tedesco (Editores). Las nuevas tecnologías y el futuro de la educación. Buenos Aires: IIPE-UNESCO/Grupo Editor.

Castillo, N. (2013). Políticas docentes en Centro América. Tendencias nacionales. Panamá. PREAL.

Coll, C., y Monereo, C. (eds) (2008). Psicología de la educación virtual. Madrid: Morata.

Dávila, A., Ruiz-Bolívar, C., y Francisco, J. (2013). Modelo tecno-pedagógico para la implantación de la modalidad semipresencial en la educación universitaria. En Revista EDUCARE, 17 (3), 115-140. Recuperado de https://revistas.investigacion-upelipb.com/index.php/educare/article/view/151

Escudero. J. (2009). Comunidades docentes de aprendizaje, formación del profesorado y mejora de la educación. En Ágora para la EF y el Deporte, 10, 7-31. Recuperado de https://dialnet.unirioja.es/ servlet/articulo?codigo $=3048583$

Fullan, M. (2002). El significado del cambio educativo: un cuarto de siglo de aprendizaje. Profesorado, revista de currículum y formación del profesorado, 6(1-2). Recuperado de https://www.ugr. es/ recfpro/rev61ART1.pdf

Gros, B. y Lara, P. (2009). Estrategias de innovación en la educación superior: el caso

de la Universitat Oberta de Catalunya. En Revista iberoamericana de educación. Pp. 223-245. Recuperado de https://doi.org/10.35362/rie490681

Hunt, B. (2008). Efectividad del desempeño docente: una reseña de la literatura internacional y su relevancia para mejorar la educación en América Latina. Santiago de Chile: PREAL.

Khan, S. (2019). Khan academic. Recuperado de https://www.khanacademy.org/

Londoño, C. (2017). Nueve tendencias de innovación educativa en América Latina. Recuperado de https:// eligeeducar.cl/9-tendencias-innovacion-educativa-america-latina.

López, C. y Heredia, Y. (2017). Escala i: Marco de referencia para la evaluación de proyectos de innovación educativa. Guía de Aplicación. Monterrey, Nuevo León: Instituto Tecnológico y de Estudios Superiores de Monterrey.

Lugo, M. (2010). Las políticas TIC en la educación de América Latina. Tendencias y experiencias. Revista Fuentes, 10, 52-68. Recuperado de https://idus.us.es/bitstream/handle/11441/32395/ Las\%20politicas\%20tic\%20en\%20la\%20educacion\%20de\%20America\%20latina. pdf?sequence $=1 \&$ isAllowed $=y$

Morin, E. (2000). Introducción al pensamiento complejo. Barcelona (España): Gedisa.

Murillo, A. (2017). ¿Qué es la innovación educativa? Recuperado de https://observatorio.tec.mx/ edu-news/innovacion-educativa

Núñez de Sarmiento, M., y Gómez, O. (2005). El factor humano: Resistencia a la innovación tecnológica. En Revista Orbis/Ciencias Humanas, Año 1(1), 2005. Recuperado de https://dialnet.unirioja.es/ servlet/articulo?codigo $=2251148$

OEI-IESME (2018). Estudio sobre la inclusión de las TIC en los centros educativos de aulas. Fundación telefónica. Madrid (España): Grafilia, S.L.

Rivas, A. (2017). Cambio e innovación educativa: Las cuestiones cruciales. XII Foro latinoamericano de educación. Fundación Santillana, Buenos Aires.

Roig-Ibáñez, J. (2006). La educación ante un nuevo orden mundial. Madrid: Ediciones Diaz de Santos. Recuperado de https://www.google.com/books/edition/_/xTO0VTEDYJQC?hl=en\&gbpv=1 
Sánchez, O. (2016). Tecnologías de la información y la comunicación aplicadas a la educación. Recuperado de https://revistas.uptc.edu.co/revistas/index.php/praxis_saber/article/view/5215.

Scardamalia, M. y Bereiter, C. (2016). Innovación y calidad: mover las Ideas hacia el centro. En Cueto, S. (ed.). Innovación y calidad en educación en América Latina. Iniciativa Latinoamericana de Investigación para las Políticas Públicas (ILAIPP) Grupo de Análisis para el Desarrollo (GRADE), Lima, Perú.

Senge, P. (2011). La Quinta disciplina. (2da ed.). Buenos Aires: Ediciones Granica.

Schein, E. (2012). La cultura organizacional: Un modelo dinámico. Barcelona (España): Plaza \& Janes Editores.

Stewart, W.H., May, R.C., McCarthy, D.J., Puffer, S.M., (2009). A test of the measurement validity of the resistance to change scale in Russia and Ukraine. The Journal of Applied Behavioral Science, 45(4), 468-489. Recuperado de https://journals.sagepub.com/doi/10.1177/0021886309338813

Tedesco, J. (2004). ¿Por qué son tan difíciles los pactos educativos? Revista Iberoamericana de Educación Organización de Estados Iberoamericanos para la Educación, la Ciencia y la Cultura (OEI). Recuperado de http://redalyc.uaemex.mx/pdf/800/80003403.pdf. Consultado 29/06/2011

Torres, R. (2005). 12 Tesis para el cambio educativo: Justicia educativa y justicia económica. En Fe y Alegría. Recuperado de http://www.feyalegria.org/?caso=5\&id=96. Consultado 2242011.

UNESCO (1990). Proyecto principal de educacion en América Latina y el Caribe. Santiago, Chile.

UNESCO (2000). Las tecnologías de la información y la comunicación en la enseñanza: Manual para docentes. Recuperado de https://unesdoc.unesco.org/ark:/48223/pf0000139028_spa

UNESCO/OREALC (2012). Antecedentes y Criterios para la Elaboración de Políticas Docentes en América Latina y el Caribe. Recuperado de https://unesdoc.unesco.org/ark:/48223/pf0000223249

UNESCO (2014). Innovación. Educativa. Serie "Herramientas de apoyo para el trabajo docente". Recuperado de http://www.cne.gob.pe/images/stories/cne-publicaciones/AvancesPEN.pdf

UNESCO (2016). Innovación educativa. Recuperado de https://unesdoc.unesco.org/ark:/48223/ pf0000247005

UNESCO/OREALC (2007). Educación de calidad para todos: un asunto de derechos humanos. Santiago: UNESCO/OREALC.

Vaillant, D. (2006). Atraer y retener buenos profesionales en la profesión docente: políticas en Latinoamérica. Revista de Educación, 340, 117-140. Recuperado de http://www.revistaeducacion. educacion.es/re340/re340_05.pdf

Para citar este documento:

Ríos-Cabrera, P. y Bolívar-Ruiz, C. (2020). La innovación educativa en América Latina: lineamientos para la formulación de políticas públicas. Revista Innovaciones Educativas. 22(32), 199212. DOI: https://doi.org/10.22458/ie.v22i32.2828 